S. Ikeda

Nagoya Math. J.

Vol. 89 (1983), 47-63

\title{
THE COHEN-MACAULAYNESS OF THE REES ALGEBRAS OF LOCAL RINGS
}

\author{
SHIN IKEDA
}

\section{§. Introduction}

Let $(A, \mathfrak{m}, k)$ be a Noetherian local ring. We define

$$
R(A)=\bigoplus_{n \geq 0} \mathfrak{m}^{n}
$$

and call it the Rees algebra of $A$. Let $X$ be an indeterminate over $A$, then $R(A)$ can be identified with the $A$-subalgebra $A[\{a X \mid a \in \mathfrak{m}\}]$ of $A[X]$. Note that the associated graded ring

$$
G(A)=\bigoplus_{n \geq 0} \mathfrak{m}^{n} / \mathfrak{m}^{n+1}
$$

of $A$ is isomorphic to $R(A) / \mathfrak{m} R(A)$.

The purpose of this paper is to give a criterion for $R(A)$ to be CohenMacaulay. For this purpose we may assume that the residue field $k=A / \mathrm{m}$ is infinite. In fact, if $k$ is finite we replace $A$ by $A[T]_{\mathrm{m} A[T]}=A(T)$, where $T$ is an indeterminate over $A$. Then $A(T)$ is faithfully flat over $A$ and is a local ring with maximal ideal $m A(T)$. Hence

$$
R(A(T)) \stackrel{\sim}{\longrightarrow} R(A) \otimes_{A} A(T)
$$

and this is Cohen-Macaulay if and only if $R(A)$ is Cohen-Macaulay. Therefore in this paper all local rings are assumed to have infinite residue field. Since $A / \mathfrak{m}$ is infinite there exist $a_{1}, a_{2}, \cdots, a_{d} \in \mathfrak{m}$ such that $\mathfrak{m}^{n}=\left(a_{1}, a_{2}, \cdots\right.$, $\left.a_{d}\right) \mathfrak{m}^{n-1}$ for some positive integer $n$, where $d=\operatorname{dim} A$ (cf. [6]), and $\mathfrak{q}=$ $\left(a_{1}, \cdots, a_{d}\right)$ is called a minimal reduction of $\mathrm{m}$.

Now we state our criterion.

Theorem (0.1). Let $(A, \mathfrak{m}, k)$ be a Noetherian local ring of dimension $d>0, \mathfrak{q}=\left(a_{1}, \cdots, a_{d}\right)$ a minimal reduction of $\mathfrak{m}$ and $\mathfrak{B}=G(A)_{+}$. Then 
the following conditions are equivalent.

1) $R(A)$ is Cohen-Macaulay.

2) a) $A$ is Buchsbaum,

b) for $i<d$

$$
\left[H_{\mathfrak{\beta}}^{i}(G(A))\right]_{n}= \begin{cases}H_{\mathfrak{m}}^{i}(A) & (n=-1) \\ (0) & (n \neq-1),\end{cases}
$$

and

c) $\mathfrak{m}^{d}=\mathfrak{q m}^{d-1}$.

In this case $G(A)_{\mathfrak{B}}$ is Buchsbaum.

Y. Shimoda proved a slightly more complicated but essentially the same result for $\operatorname{dim} A=3$ and he communicated it to me informally. I have also learned that $\mathrm{S}$. Goto independently obtained the implication 1 ) $\Rightarrow 2$ ).

Our theorem generalizes a result due to S. Goto and Y. Shimoda which says that when $A$ is Cohen-Macaulay, $R(A)$ is Cohen-Macaulay if and only if $G(A)$ is Cohen-Macaulay and there exist $a_{1}, \cdots, a_{d} \in \mathfrak{m}$ such that $\mathfrak{m}^{d}=$ $\left(a_{1}, \cdots, a_{d}\right) \mathfrak{m}^{d-1}$ (cf. [2]). Our proof of the condition $\mathrm{c}$ ) is the same as that of [2].

The theory of Buchsbaum rings has been rapidly developed in recent years as a generalization of that of Cohen-Macaulay rings. It is remarkable that the theory of Buchsbaum rings can be related to the CohenMacaulayness of the Rees algebra as our result shows.

In section 1 we recall the definition of Buchsbaum rings and their basic properties which we need in this paper. Section 2 is devoted to the proof of Theorem (0.1). The idea of the proof was inspired by the techniques of S. Goto. The proof is carried out by detailed computations of local cohomology modules. In section 3 we give an example of a Buchsbaum ring which is not Cohen-Macaulay and whose Rees algebra is CohenMacaulay.

All rings in this paper are commutative with identity.

Before closing this section the author would like to thank S. Goto and J. Watanabe for helpful advices and inspiring discussions.

\section{§1. Buchsbaum rings}

Recall the definition of Buchsbaum rings. Let $(A, \mathfrak{m}, k)$ be a Noetherian local ring and $M$ a finitely generated $A$-module. An ideal of $A$ is called 
a parameter ideal of $M$ if it is generated by a system of parameters of $M$.

We say that $M$ is a Buchsbaum $A$-module if for any parameter ideal $\mathfrak{q}$ of $M$ the difference $1_{A}(M / \mathfrak{q} M)-e_{M}(\mathfrak{q})$ is an invariant of $M$ which does not depend on the choice of the parameter ideal $q$ of $M$, where $1_{A}()$ denotes the length of $A$-module and $e_{M}(\mathfrak{q})$ is the multiplicity of $q$ with respect to $M$. We denote this invariant of $M$ by $I(M)$. A Noetherian local ring is Buchsbaum if it is a Buchsbaum module over itself.

Before recalling the basic properties of Buchsbaum rings we introduce some notation. Let $R$ be a Noetherian ring, $M$ a finitely generated $R$-module and $N$ a proper submodule of $M$. Let $\operatorname{Assh}_{R}(M / N)=\left\{\mathfrak{p} \in \operatorname{Supp}_{R}(M / N)\right\}$ $\operatorname{dim} R / p=\operatorname{dim} M / N\}$. Let $N=\bigcap_{\mathfrak{p} \in \operatorname{Assh}_{R}(M / N)} N(\mathfrak{p})$ be a primary decomposition of $N$ in $M$, where $N(\mathfrak{p})$ is a $p$-primary submodule of $M$. Then we define

$$
U(N)=\bigcap_{\mathfrak{p} \in \operatorname{Assh}_{R}(M / N)} N(\mathfrak{p}) .
$$

Note that $U(N)$ does not depend on the primary decomposition chosen since every $\mathfrak{p} \in \operatorname{Assh}_{R}(M / N)$ is minimal in $\operatorname{Supp}_{R}(M / N)$.

Let $R$ be as above and $\mathfrak{a}$ an ideal of $R$. For any $R$-module $M$ we define

$$
H_{\mathrm{a}}^{i}(M)=\underset{n}{\lim } \operatorname{Ext}_{R}^{i}\left(R / \mathfrak{a}^{n}, M\right)
$$

and call it the $i$-th local cohomology module of $M$ with support in Spec $R / \mathfrak{a}$. The functor $H_{\mathrm{a}}^{i}()$ is the $i$-th derived functor of $H_{\mathrm{a}}^{0}()$. If $R=\oplus_{n \geq 0} R_{n}$ is a graded ring, a a homogeneous ideal of $R$ and $M=\oplus_{n \in Z} M_{n}$ a graded $R$-module, then $H_{a}^{i}(M)$ has a natural structure of graded $R$-module. We denote the homogeneous part of degree $n$ of $H_{\mathrm{a}}^{i}(M)$ by $\left[H_{\mathrm{a}}^{i}(M)\right]_{n}$.

Now let us recall the basic properties of Buchsbaum rings.

Proposition (1.1). Let $(A, \mathfrak{m}, k)$ be a Noetherian local ring of dimension $d>0$. Then the following conditions are equivalent.

1) $A$ is Buchsbaum.

2) For any system of parameters $a_{1}, a_{2}, \cdots, a_{d}$ of $A$, we have

$$
\mathfrak{m} U\left(\left(a_{1}, a_{2}, \cdots, a_{i}\right)\right) \subset\left(a_{1}, a_{2}, \cdots, a_{i}\right) \text { for } 0 \leq i<d .
$$

Proof. See [10].

Corollary (1.2). Let $(A, \mathrm{~m}, k)$ be a Buchsbaum ring of dimension $d>0$. Then, 
1) for any $a \in \mathfrak{m}$ such that $\operatorname{dim} A / a A<\operatorname{dim} A, A / a A$ is Buchsbaum,

2) for any system of parameters of $A$, $U\left(\left(U\left(\left(a_{1}, a_{2}, \cdots, a_{i}\right)\right), a_{i+1}\right)\right)=U\left(\left(a_{1}, a_{2}, \cdots, a_{i+1}\right)\right) \quad$ for $0 \leq i \leq d-2$,

3) $A / U((0))$ is Buchsbaum, and

4) $U\left(\left(a_{1}, a_{2}, \cdots, a_{i}\right)\right)=\left(a_{1}, a_{2}, \cdots, a_{i}\right): a_{i+1}$ for $0 \leq i<d$.

Proof. See [10].

Proposition (1.3). Let $(A, \mathfrak{m}, k)$ be a Noetherian local ring and $\hat{A}$ the completion of $A$. Then $A$ is Buchsbaum if and only if $\hat{A}$ is Buchsbaum.

Proof. See [9].

Proposition (1.4). Let $(A, \mathfrak{m}, k)$ be a Buchsbaum ring of dimension $d>0$. Then,

1) $\mathfrak{m} H_{\mathfrak{m}}^{i}(A)=(0)$ for $0 \leq i<d$, and

2) $I(A)=\sum_{i=0}^{d-1}\left(\begin{array}{c}d-1 \\ i\end{array}\right) \operatorname{dim}_{k} H_{\mathrm{m}}^{i}(A)$, where $\operatorname{dim}_{k}$ denotes the dimension of $k$-vector space.

Proof. See [8] or [3].

Proposition (1.5). Let $(A, \mathfrak{m}, k)$ be a Noetherian local ring and $M a$ finitely generated A-module of dimension $d>0$. Assume that the canonical homomorphism

$$
\phi_{i}: \operatorname{Ext}_{A}^{i}(k, M) \longrightarrow H_{\mathfrak{m}}^{i}(M)=\underset{n}{\lim } \operatorname{Ext}_{A}^{i}\left(A / \mathfrak{m}^{n}, M\right)
$$

is surjective for $0 \leq i<d$. Then $M$ is a Buchsbaum A-module. Furthermore if $A$ is regular the converse is true.

Proof. See [11].

Proposition (1.6). Let $k$ be a field, $G=\oplus_{n \geq 0} G_{n}$ a Noetherian graded ring with $G_{0}=k$ and $\mathfrak{P}=G_{+}$. Suppose that there is an integer $n$ such that for $0 \leq i<d=\operatorname{dim} G$ and $m \neq n$

$$
\left[H_{\mathfrak{R}}^{i}(G)\right]_{m}=(0) \text {. }
$$

Then $G_{\Re}$ is Buchsbaum.

Proof. See [8].

The following result is crucial in section 2 . 
Proposition (1.7). Let $(A, \mathfrak{m}, k)$ be a Buchsbaum ring of dimension $d>0, G=G(A)$ and $\mathfrak{\beta}=G_{+}$. If $G_{\Re}$ is Buchsbaum and $I(A)=I\left(G_{\Re}\right)$, then for any minimal reduction $\mathfrak{q}=\left(a_{1}, a_{2}, \cdots, a_{d}\right)$ of $\mathfrak{m}$ we have

$$
\left(a_{1}^{n_{1}}, a_{2}^{n_{2}}, \cdots, a_{d}^{n_{d}}\right) \cap \mathfrak{m}^{n}=\sum_{i=1}^{d} a_{i}^{n_{i}} \mathfrak{m}^{n-n_{i}}
$$

for all positive integers $n_{1}, n_{2}, \cdots, n_{d}, n$, where $\mathfrak{m}^{j}=A$ if $j \leq 0$.

Proof. Let $h_{i}(1 \leq i \leq d)$ be the initial form of $a_{i}$ in $G$. Then $h_{1}$, $h_{2}, \cdots, h_{d}$ is a system of parameters of $G_{\mathfrak{r}}$. By the definition of Buchsbaum rings, we have

$$
\begin{aligned}
& 1_{G}\left(G /\left(h_{1}^{n_{1}} h_{2}^{n_{2}}, \cdots, h_{d}^{n_{d}}\right)\right)-e_{G}\left(\left(h_{1}^{n_{1}}, h_{2}^{n_{2}}, \cdots, h_{d}^{n_{d}}\right)\right) \\
& \quad=1_{A}\left(A /\left(a_{1}^{n_{1}}, a_{2}^{n_{2}}, \cdots, a_{d}^{n_{d}}\right)\right)-e_{A}\left(\left(a_{1}^{n_{1}}, a_{2}^{n_{2}}, \cdots, a_{d}^{n_{d}}\right)\right)
\end{aligned}
$$

for all positive integers $n_{1}, n_{2}, \cdots, n_{d}$.

So we have

$$
1_{G}\left(G /\left(h_{1}^{n_{1}}, h_{2}^{n_{2}}, \cdots, h_{d}^{n_{d}}\right)\right)=1_{A}\left(A /\left(a_{1}^{n_{1}}, a_{2}^{n_{2}}, \cdots, a_{d}^{n_{d}}\right)\right)
$$

because

$$
e_{G}\left(\left(h_{1}^{n_{1}}, \cdots, h_{d}^{n_{d}}\right)\right)=n_{1} n_{2} \cdots n_{d} e_{G}\left(\left(h_{1}, \cdots, h_{d}\right)\right)
$$

and

$$
e_{A}\left(\left(a_{1}^{n_{1}}, \cdots, a_{d}^{n_{d}}\right)\right)=n_{1} n_{2} \cdots n_{d} e_{A}\left(\left(a_{1}, \cdots, a_{d}\right)\right)
$$

by [1] and because

$$
e_{G}\left(\left(h_{1}, \cdots, h_{d}\right)\right)=e_{A}\left(\left(a_{1}, \cdots, a_{d}\right)\right) .
$$

On the other hand,

$$
1_{G}\left(G /\left(h_{1}^{n_{1}}, \cdots, h_{d}^{n_{d}}\right)\right)=\sum_{n \geq 0} 1_{A}\left(\mathfrak{m}^{n} / \sum_{i=1}^{d} a_{i}^{n_{i}} \mathfrak{m}^{n-n_{i}}+\mathfrak{m}^{n+1}\right)
$$

and

$$
1_{A}\left(A /\left(a_{1}^{n_{1}}, \cdots, a_{d}^{n_{d}}\right)\right)=\sum_{n \geq 0} 1_{A}\left(\mathfrak{m}^{n} /\left(a_{1}^{n_{1}}, \cdots, a_{d}^{n_{d}}\right) \cap \mathfrak{m}^{n}+\mathfrak{m}^{n+1}\right)
$$

But

$$
\sum_{i=1}^{d} a_{i}^{n_{i}} \mathfrak{m}^{n-n_{i}}+\mathfrak{m}^{n+1} \subset\left(a_{1}^{n_{1}}, \cdots, a_{d}^{n_{d}}\right) \cap \mathfrak{m}^{n}+\mathfrak{m}^{n+1}
$$

for all $n>0$. Hence, 


$$
\sum_{i=1}^{d} a_{i}^{n_{i}} \mathfrak{m}^{n-n_{i}}+\mathfrak{m}^{n+1}=\left(a_{1}^{n_{1}}, \cdots, a_{d}^{n d}\right) \cap \mathfrak{m}^{n}+\mathfrak{m}^{n+1}
$$

for all positive integers $n_{1}, n_{2}, \cdots, n_{d}, n$.

Therefore

$$
\sum_{i=1}^{d} a_{i}^{n_{i}} \mathfrak{m}^{n-n_{i}}=\left(a_{1}^{n_{1}}, \cdots, a_{d}^{n_{d}}\right) \cap \mathfrak{m}^{n}
$$

for all positive integers $n_{1}, n_{2}, \cdots, n_{d}, n$.

Corollary (1.8). Under the same hypothesis as in (1.7) we have

$$
\left(a_{1}^{n_{1}}, \cdots, a_{i}^{n_{i}}\right) \cap \mathfrak{m}^{n}=\sum_{j=1}^{i} a_{j}^{n_{j}} \mathfrak{m}^{n-n_{j}}
$$

for $1 \leq i \leq d$ and for all positive integers $n_{1}, n_{2}, \cdots, n_{i}, n$.

Proof.

$$
\begin{aligned}
\left(a_{1}^{n_{1}}, \cdots, a_{i}^{n_{i}}\right) \cap \mathfrak{m}^{n} & =\left(\bigcap_{k>0}\left(a_{1}^{n_{1}}, \cdots, a_{i}^{n_{i}}, a_{i+1}^{k}, \cdots, a_{d}^{k}\right)\right) \cap \mathfrak{m}^{n} \\
& =\bigcap_{k>0}\left(\left(a_{1}^{n_{1}}, \cdots, a_{i}^{n_{i}}, a_{i+1}^{k}, \cdots, a_{d}^{k}\right) \cap \mathfrak{m}^{n}\right) \\
& =\bigcap_{k>0}\left(\sum_{j=1}^{i} a_{j}^{n_{j}} \mathfrak{m}^{n_{-n_{j}}},\left(a_{i+1}^{k}, \cdots, a_{d}^{k}\right) \mathfrak{m}^{n-k}\right) \\
& =\sum_{j=1}^{i} a_{j}^{n_{j}} \mathfrak{m}^{n_{-n}} .
\end{aligned}
$$

\section{§2. Proof of Theorem (0.1)}

Let $(A, \mathrm{~m}, k)$ be a Noetherian local ring of dimension $d>0, R=R(A)$ and $G=G(A)$. Let $X$ be an indeterminate over $A$. We identify $R$ with the graded $A$-subalgebra $A[\{a X \mid a \in \mathfrak{m}\}]$ of $A[X]$. Then the homogeneous component of degree $n$ of $R$ is given by $[R]_{n}=\mathfrak{m}^{n} X^{n}$ for $n \geq 0$ and $[R]_{n}$ $=(0)$ for $n<0$. Let $R_{+}=\oplus_{n>0}[R]_{n}$ and let

$$
h: R \longrightarrow A=R / R_{+}
$$

be the canonical projection. Let $E$ be an $A$-module. We denote $E$ by ${ }_{n} E$ if $E$ is considered as an $R$-module via $h$. Furthermore ${ }_{h} E$ is considered as a graded $R$-module whose grading is given by $\left[{ }_{h} E\right]_{0}=E$ and $\left[{ }_{h} E\right]_{n}=$ (0) for $n \neq 0$. Let $M$ be the unique maximal homogeneous ideal of $R$, then

$$
H_{M}^{i}\left({ }_{h} E\right)={ }_{h} H_{m}^{i}(E) \quad \text { for all } i \geq 0 \quad \text { (cf. [4]) . }
$$


Now recall that $\operatorname{dim} R=\operatorname{dim} R_{M}=d+1$ (cf. [12]). Let $\mathfrak{q}=\left(a_{1}, a_{2}, \cdots\right.$, $a_{d}$ ) be a minimal reduction of $\mathfrak{m}$ and we set

$$
Q=\left(a_{1}, a_{2}-a_{1} X, \cdots, a_{d}-a_{d-1} X, a_{d} X\right) .
$$

LEMMA (2.1). $Q R_{M}$ is a minimal reduction of $M R_{M}$. In particular $a_{1}$, $a_{2}-a_{1} X, \cdots, a_{d}-a_{d-1} X, a_{d} X$ is a system of parameters of $R_{M}$.

Proof. Let $P=\left(a_{1}, a_{2}, \cdots, a_{d}, a_{1} X, a_{2} X, \cdots, a_{d} X\right)$ and $n$ a positive integer such that $\mathfrak{m}^{n}=\mathfrak{q m}^{n-1}$. Then,

$$
M^{n}=P M^{n-1} .
$$

But it is easy to see that $P^{2}=Q P$. Hence

$$
M^{n+1}=P^{2} M^{n-1}=Q P M^{n-1}=Q M^{n} .
$$

Lemma (2.2). $R$ is Cohen-Macaulay if and only if $a_{1}, a_{2}-a_{1} X, \cdots, a_{d}$ $-a_{d-1} X, a_{d} X$ is an $R_{M}$-sequence.

Proof. By [5] $R$ is Cohen-Macaulay if and only if $R_{M}$ is CohenMacaulay. Hence (2.2) follows from (2.1).

We first prove 1$) \Rightarrow 2$ ).

Suppose $R$ is Cohen-Macaulay. Then $a_{1}, a_{2}-a_{1} X, \cdots, a_{d}-a_{d-1} X$, $a_{d} X$ is an $R_{M}$-sequence by (2.2). In particular $a_{1}$ is a non-zero divisor on $A$. For any $x \in \mathfrak{m}^{d}$,

$$
\begin{aligned}
a_{1} x X^{d} & \equiv a_{2} x X^{d-1} \\
& \equiv a_{3} x X^{d-2} \\
& \vdots \\
& \equiv a_{d} x X \\
& \equiv 0 \bmod \left(a_{2}-a_{1} X, \cdots, a_{d}-a_{d-1} X, a_{d} X\right) .
\end{aligned}
$$

Hence we can find $g \in R$ not contained in $M$ such that

$$
g\left(x X^{d}\right)=\left(a_{2}-a_{1} X\right) f_{1}+\cdots+\left(a_{d}-a_{d-1} X\right) f_{d-1}+a_{d} X f_{d}
$$

for some $f_{1}, f_{2}, \cdots, f_{d} \in R$. Comparing the coefficients of $X^{d}$ in the equation (\#), we have $x \in \mathfrak{q m}^{d-1}$ since the constant term of $g$ is a unit of $A$. Thus $\mathfrak{m}^{d}=\mathfrak{q m}^{d-1}$ and this shows c). The above method of the proof is the same as that of [2].

We need an easy lemma to prove a) and b).

Lemma (2.3). Let $a \in \mathfrak{m}$ be a non-zero divisor on $A$. Then we have the 
following exact sequences of graded R-modules

$$
\begin{aligned}
& 0 \longrightarrow G(-1) \longrightarrow R / a R \longrightarrow R /(a, a X) R \longrightarrow 0 \\
& 0 \longrightarrow R /(a X R \longrightarrow R /(a, a X) R \longrightarrow 0 .
\end{aligned}
$$

Proof. Consider the exact sequences of graded $R$-modules

$$
\begin{aligned}
& 0 \longrightarrow(a, a X) R / a R \longrightarrow R / a R \longrightarrow R /(a, a X) R \longrightarrow 0 \\
& 0 \longrightarrow(a, a X) R / a X R \longrightarrow R / a X R \longrightarrow R /(a, a X) R \longrightarrow 0 .
\end{aligned}
$$

But we have the isomorphisms of graded $R$-modules

$$
\begin{aligned}
& (a, a X) R / a R \stackrel{\sim}{\longrightarrow} a X R / a X(a R: a X) \stackrel{\sim}{\longrightarrow} R /(a R: a X)(-1) \\
& (a, a X) R / a X R \stackrel{\sim}{\longrightarrow} a R / a(a X R: a) \stackrel{\sim}{\longrightarrow} /(a X: a) .
\end{aligned}
$$

Since $a$ is a non-zero divisor we have $(a R: a X)=\mathfrak{m} R$ and $(a X R: a)=R_{+}$. Therefore we have the exact sequences of graded $R$-modules

$$
\begin{aligned}
& 0 \longrightarrow G(-1) \longrightarrow R / a R \longrightarrow R /(a, a X) R \longrightarrow 0 \\
& 0 \longrightarrow{ }_{h} A \longrightarrow R / a X R \longrightarrow R /(a, a X) R \longrightarrow 0 .
\end{aligned}
$$

Let $a=a_{1}$ and let $R^{\prime}=R /(a, a X) R$. Then we have the exact sequences of graded $R$-modules

$$
\begin{aligned}
& 0 \longrightarrow G(-1) \longrightarrow R / a R \longrightarrow R^{\prime} \longrightarrow 0 \\
& 0 \longrightarrow{ }_{h} A \longrightarrow R / a X R \longrightarrow R^{\prime} \longrightarrow 0
\end{aligned}
$$

from (2.3).

Since $a$ is a non-zero divisor on $A R / a R$ and $R / a X R$ are CohenMacaulay rings of dimension $d$. From the exact sequences $(*)$ and $(* *)$ we have the isomorphisms of graded $R$-modules,

$$
H_{M}^{i-1}\left(R^{\prime}\right) \stackrel{\sim}{\longrightarrow} H_{M}^{i}(G)(-1) \text { and } H_{M}^{i}\left(R^{\prime}\right) \stackrel{\sim}{\longrightarrow} H_{M}^{i-1}\left({ }_{h} A\right)={ }_{h} H_{\mathrm{m}}^{i}(A)
$$

But by [5]

$$
H_{M}^{i}(G)=H_{\Re}^{i}(G) \quad \text { for all } i, \quad \text { where } \mathfrak{P}=G_{+} .
$$

Thus, we have for $i<d$

$$
\left[H_{\aleph}^{i}(G)\right]_{n}=\left\{\begin{array}{cc}
H_{\mathrm{m}}^{i}(A) & (n=-1) \\
(0) & (n \neq-1) .
\end{array}\right.
$$

Therefore $G_{\mathfrak{p}}$ is Buchsbaum by (1.6). This shows b). 
To prove a) we may assume $A$ is complete by (1.3). Let $(B, \mathfrak{n}, k)$ be a regular local ring such that $A$ is a homomorphic image of it. Let $X_{1}$, $X_{2}, \cdots, X_{v}$ be indeterminates over $B$, where $v=\operatorname{emb} A$, the embedding dimension of $A$, and we set $S=B\left[X_{1}, X_{2}, \cdots, X_{v}\right]$. We give $S$ a structure of graded $B$-algebra by letting $\operatorname{deg} X_{i}=1(1 \leq i \leq v)$. Let $N=\left(\mathfrak{n}, X_{1}, \cdots, X_{v}\right)$ be the unique maximal homogeneous ideal of $S$. We can choose $a_{d+1}, \cdots$, $a_{v} \in \mathfrak{m}$ so that $\mathfrak{m}=\left(a_{1}, \cdots, a_{d}, a_{d+1}, \cdots, a_{v}\right)$ by [6]. We define a surjective homomorphism of graded $B$-algebra $\alpha: S \rightarrow R$ by $\alpha\left(X_{i}\right)=a_{i} X(1 \leq i \leq v)$. Since $G \widetilde{\rightrightarrows} R / \mathrm{m} R$ and $A \widetilde{\rightrightarrows} R / R_{+}$both $G$ and $A$ are homomorphic images of $S$. By the definition of Buchsbaum rings $G_{\mathfrak{B}}$ (resp. $A$ ) is Buchsbaum if and only if $G_{\Re}$ (resp. $A$ ) is a Buchsbaum $S_{N}$-module. Let $T=S_{N}$ and $\mathfrak{a}=N S_{N}$. Then, from the exact sequences $(*)$ and $(* *)$ we get the following commutative diagrams of $T$-modules

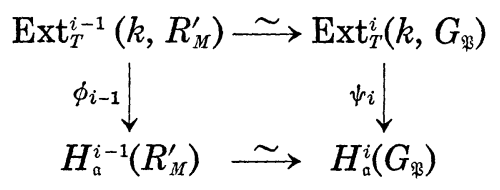

and

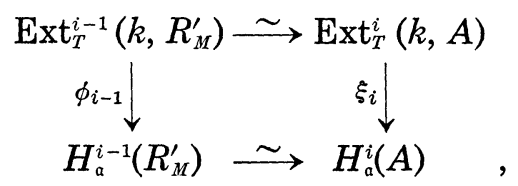

where $i<d$ and $\phi_{i-1}, \psi_{i}$ and $\xi_{i}$ are the canonical homomorphisms. Since $T$ is regular local and $G_{\mathfrak{r}}$ is Buchsbaum $\psi_{i}$ is surjective for $i<d$ by (1.5). Hence $\xi_{i}$ is surjective for $i<d$ by the commutative diagrams above. Therefore $A$ is Buchsbaum by (1.5). This completes the proof of 1) $\Rightarrow 2$ ).

Conversely, assume that 2) holds.

First we set for $0 \leq i<d$

$$
\begin{aligned}
\mathfrak{q}_{i} & =\left(a_{1}, \cdots, a_{i}\right), \\
A_{i} & =A / U\left(\mathfrak{q}_{i}\right), \\
R_{i} & =R\left(A_{i}\right)
\end{aligned}
$$

and

$$
G_{i}=G\left(A_{i}\right),
$$

where $\mathfrak{q}_{0}=(0)$. Let $i, j$ be integers. Then we set $[i, j]=\{n \in Z \mid i \leq n \leq j\}$. 
Lemma (2.4). Let $h_{i}(1 \leq i \leq d)$ be the initial form of $a_{i}$ in $G$. Then, for $0 \leq i<d$ and $0 \leq j<d-i$ we have

$$
\left[H_{\Re}^{j}\left(G /\left(h_{1}, \cdots, h_{i}\right)\right)\right]_{n}=(0) \quad \text { for } n \notin[-1, i-1] \text {. }
$$

Proof. For $i=0$, there is nothing to prove. We proceed by induction on $i$. Assume $i>0$. Since $G_{\mathfrak{p}}$ is Buchsbaum we have the following exact sequence of graded $G$-modules

$$
\begin{aligned}
0 \longrightarrow H_{\mathfrak{B}}^{j}\left(G /\left(h_{1}, \cdots, h_{i-1}\right)\right) \longrightarrow H_{\mathfrak{\beta}}^{j}\left(G /\left(h_{1}, \cdots, h_{1}\right)\right) \\
\longrightarrow H_{\mathfrak{B}}^{j+1}\left(G /\left(h_{1}, \cdots, h_{i-1}\right)\right)(-1) \longrightarrow 0
\end{aligned}
$$

for $0 \leq j<d-i$. By the induction hypothesis

$$
\left[H_{\mathfrak{P}}^{j}\left(G /\left(h_{1}, \cdots, h_{i-1}\right)\right)\right]_{n}=(0)
$$

for $0 \leq j \leq d-i$ and $n \notin[-1, i-2]$.

Hence we have

$$
\left[H_{\Re}^{j}\left(G /\left(h_{1}, \cdots, h_{i}\right)\right)\right]_{n}=(0)
$$

for $0 \leq j<d-i$ and $n \notin[-1, i-1]$.

Lemma (2.5). $\quad U\left(\mathfrak{q}_{i}\right) \cap \mathfrak{m}^{n}=\mathfrak{q}_{i} \mathfrak{m}^{n-1}$ for $0 \leq i<d$ and $n \geq i$.

Proof. By (2.4)

$$
\left[H_{\mathfrak{\beta}}^{0}(G)\right]_{n}=(0)
$$

for $n \neq-1$. Hence

$$
H_{\Re}^{0}(G)=(0) .
$$

Since $G_{\Re}$ is Buchsbaum this means

$$
\left((0): h_{i}\right)_{G}=((0): \mathfrak{P})_{G}=(0)
$$

for $1 \leq i \leq d$. Hence

$$
\left(\mathfrak{m}^{n}: a_{i}\right)=\mathfrak{m}^{n-1}
$$

for $1 \leq i \leq d$ and $n>0$. By (1.2) $G_{\Re} /\left(h_{1}, \cdots, h_{i}\right) G_{\Re}$ is Buchsbaum for $1 \leq$ $i \leq d$. Therefore

$$
H_{\Re}^{0}\left(G /\left(h_{1}, \cdots, h_{i}\right)\right)=\left(\left(h_{1}, \cdots, h_{i}\right): h_{i+1}\right)_{G} /\left(h_{1}, \cdots, h_{i}\right)
$$

for $0 \leq i<d$. By (2.4)

$$
\left[H_{\mathfrak{P}}^{0}\left(G /\left(h_{1}, \cdots, h_{i}\right)\right)\right]_{n}=(0)
$$


for $n \notin[-1, i-1]$, and this implies

$$
\left(\left(\mathfrak{q}_{i} \mathfrak{m}^{n}+\mathfrak{m}^{n+2}\right): a_{i+1}\right) \cap \mathfrak{m}^{n} \subset \mathfrak{q}_{i} \mathfrak{m}^{n-1}+\mathfrak{m}^{n+1}
$$

for $n \geq i$. On the other hand, by b) and (1.4) $I\left(G_{\Re}\right)=I(A)$. Hence by (1.8) $\mathfrak{q}_{i} \cap \mathfrak{m}^{n}=\mathfrak{q}_{i} \mathfrak{m}^{n-1}$ for $n>0$. Therefore

$$
\left(\left(\mathfrak{q}_{i} \cap \mathfrak{m}^{n+1}\right): a_{i+1}\right) \cap \mathfrak{m}^{n} \subset \mathfrak{q}_{i} \mathfrak{m}^{n-1}+\mathfrak{m}^{n+1}
$$

for $n \geq i$. By what we have just proved above $\left(\mathfrak{m}^{n+1}: a_{i+1}\right)=\mathfrak{m}^{n}$ for $n \geq 0$. Thus we have

$$
U\left(\mathfrak{q}_{i}\right) \cap \mathfrak{m}^{n}=\mathfrak{q}_{i} \mathfrak{m}^{n-1}+\mathfrak{m}^{n+1}
$$

for $n \geq i$. This yields

$$
U\left(\mathfrak{q}_{i}\right) \cap \mathfrak{m}^{n}=\mathfrak{q}_{i} \mathfrak{m}^{n-1}
$$

for $n \geq i$.

Corollary (2.6). $\quad U\left(a_{i+1} A_{i}\right) \cap \mathfrak{m}^{n} A_{i}=a_{i+1} \mathfrak{m}^{n-1} A_{i}$ for $0 \leq i \leq d-2$ and $n \geq i+1$.

Proof. It is sufficient to prove that

$$
\left(\left(U\left(\mathfrak{q}_{i}\right), a_{i+1}\right): \mathfrak{m}\right) \cap\left(\mathfrak{m}^{n}, U\left(\mathfrak{q}_{i}\right)\right)=\mathfrak{q}_{i+1} \mathfrak{m}^{n-1}+U\left(\mathfrak{q}_{i}\right)
$$

for $n \geq i+1$.

But this follows from (1.2) and (2.5).

Lemma (2.7). We have the exact sequence of graded $R$-modules

$$
{ }_{n} H_{\mathrm{m}}^{j}\left(U\left(a_{i+1} A_{i}\right)\right) \longrightarrow H_{M}^{j}\left(R_{i} / a_{i+1} X R_{i}\right) \longrightarrow H_{M}^{j}\left(R_{i+1}\right)
$$

for $0 \leq i \leq d-1$ and $j>0$.

Proof. There exist canonical surjective homomorphisms of graded $R$ modules $u_{i}: R_{i} / a_{i+1} X R_{i} \rightarrow R_{i+1}$. Let $K_{i}=\operatorname{Ker} u_{i}$. Since $A_{i+1} \underset{\rightarrow}{\rightarrow} / U\left(a_{i+1} A_{i}\right)$ by (1.2)

$$
R_{i+1} \stackrel{\sim}{\longrightarrow} \bigoplus_{n \geq 0} \mathfrak{m}^{n} A_{i} / U\left(a_{i+1} A_{i}\right) \cap \mathfrak{m}^{n} A_{i} .
$$

Hence $\left[K_{i}\right]_{0}=U\left(a_{i+1} A_{i}\right)$ and $\left[K_{i}\right]_{n}=U\left(a_{i+1} A_{i}\right) \cap \mathfrak{m}^{n} A_{i} / a_{i+1} \mathfrak{m}^{n-1} A_{i}$ for $n>0$. But $A_{i}$ is Buchsbaum by (1.2), so $\mathrm{m} U\left(a_{i+1} A_{i}\right) \subset a_{i+1} A_{i}$ by (1.1). Therefore $1_{A}\left(\left[K_{i}\right]_{n}\right)<\infty$ for $n>0$ and $\left[K_{i}\right]_{n}=(0)$ for $n \geq i+1$ by (2.6). It follows that 


$$
K_{i+}=\bigoplus_{n \geq 1}\left[K_{i}\right]_{n}
$$

is a graded $R$-module of dimension 0 . Hence from the exact sequence

$$
0 \longrightarrow K_{i+} \longrightarrow K_{i} \longrightarrow{ }_{n} U\left(a_{i+1} A_{i}\right) \longrightarrow 0,
$$

we get the isomorphism of graded $R$-modules

$$
H_{i r}^{j}\left(K_{i}\right) \stackrel{\sim}{\longrightarrow}{ }_{h} H_{\mathrm{m}}^{j}\left(U\left(a_{i+1} A_{i}\right)\right)
$$

for $j>0$. On the other hand, from the exact sequence

$$
0 \longrightarrow K_{i} \longrightarrow R_{i} / a_{i+1} X R_{i} \longrightarrow R_{i+1} \longrightarrow 0,
$$

we get the exact sequence of graded $R$-modules

$$
H_{M}^{j}\left(K_{i}\right) \longrightarrow H_{M}^{j}\left(R_{i} / a_{i+1} X R_{i}\right) \longrightarrow H_{M}^{j}\left(R_{i+1}\right)
$$

for $j>0$, and this completes the proof of (2.7).

Lemma (2.8). We have the exact sequence of graded R-modules

$$
H_{M}^{j}\left(G_{i}\right)(-1) \longrightarrow H_{M}^{j}\left(R_{i} / a_{i+1} R_{i}\right) \longrightarrow H_{M}^{j}\left(R_{i+1}\right)
$$

for $0 \leq i \leq d-2$ and $j>0$.

Proof. Let $v_{i}: R_{i} / a_{i+1} R_{i} \longrightarrow R_{i+1}$ be the canonical surjective homomorphism and $L_{i}=\operatorname{Ker} v_{i}$. Then $\left[L_{i}\right]_{n} \simeq U\left(a_{i+1} A_{i}\right) \cap \mathfrak{m}^{n} A_{i} / a_{i+1} \mathfrak{m}^{n} A_{i}$ for $n \geq 0$. On the other hand,

$$
\left[\left(a_{i+1}, a_{i+1} X\right) R_{i} / a_{i+1} R_{i}\right]_{n}=a_{i+1} \mathfrak{m}^{n-1} A_{i} / a_{i+1} \mathfrak{m}^{n} A_{i}
$$

for $n \geq 0$. Hence we have an exact sequence of graded $R$-modules

$$
0 \longrightarrow\left(a_{i+1}, a_{i+1} X\right) R_{i} / a_{i+1} R_{i} \longrightarrow L_{i} \longrightarrow L_{i}^{\prime} \longrightarrow 0,
$$

where

$$
L_{i}^{\prime}=\underset{n \geq 0}{\oplus} U\left(a_{i+1} A_{i}\right) \cap \mathfrak{m}^{n} A_{i} / a_{i+1} \mathfrak{m}^{n-1} A_{i} .
$$

By (2.6) $\operatorname{dim} L_{i}^{\prime}=0$. Since $a_{i+1}$ is a non-zero divisor on $A_{i}$ we have an isomorphism $\left(a_{i+1}, a_{i+1} X\right) R_{i} / a_{i+1} R_{i} \leftrightharpoons G_{i}(-1)$. Hence we have the exact sequences of graded $R$-modules

$$
0 \longrightarrow G_{i}(-1) \longrightarrow L_{i} \longrightarrow L_{i}^{\prime} \longrightarrow 0
$$

and

$$
0 \longrightarrow L_{i} \longrightarrow R_{i} / a_{i+1} R_{i} \longrightarrow R_{i+1} \longrightarrow 0 \text {. }
$$

Since $\operatorname{dim} L_{i}^{\prime}=0$ we have a surjective homomorphism $H_{\Re}^{1}\left(G_{i}\right)(-1) \rightarrow H_{M}^{1}\left(L_{i}\right)$ 
and an isomorphism $H_{\mathfrak{R}}^{j}\left(G_{i}\right)(-1) \widetilde{\rightarrow} H_{M}^{j}\left(L_{i}\right)$ for $j>1$. Therefore we have the exact sequence of graded $R$-modules

$$
H_{M}^{j}\left(G_{i}\right)(-1) \longrightarrow H_{M}^{j}\left(R_{i} / a_{i+1} R_{i}\right) \longrightarrow H_{M}^{j}\left(R_{i+1}\right)
$$

for $j>0$.

Lemma (2.9). We have the isomorphism of graded $R$-modules

$$
H_{M}^{j}\left(G_{i}\right) \stackrel{\sim}{\longrightarrow} H_{M}^{j}\left(G /\left(h_{1}, \cdots, h_{i}\right)\right)
$$

for $0 \leq i \leq d-2$ and $j>0$.

Proof. There is a surjective homomorphism $w_{i}: G /\left(h_{1}, \cdots, h_{i}\right) \rightarrow G_{i}$ with kernel $N_{i}=\oplus_{n \geq 0} U\left(\mathfrak{q}_{i}\right) \cap \mathfrak{m}^{n} / \mathfrak{q}_{i} \mathfrak{m}^{n-1}+U\left(\mathfrak{q}_{i}\right) \cap \mathfrak{m}^{n+1}$. By (2.5), we have $\operatorname{dim} N_{i}=0$. Hence we have the isomorphism

$$
H_{M}^{j}\left(G /\left(h_{1}, \cdots, h_{i}\right)\right) \stackrel{\sim}{\longrightarrow} H_{M}^{j}\left(G_{i}\right)
$$

for $j>0$.

Lemma (2.10). $\left[H_{M}^{0}\left(R_{i} / a_{i+1} R_{i}\right)\right]_{n}=(0)$ for $0 \leq i<d$ and $n \notin[0, i-1]$.

Proof. By the definition of local cohomology, we have

$$
H_{M}^{0}\left(R_{i} / a_{i+1} R_{i}\right) \stackrel{\sim}{\longrightarrow}\left(a_{i+1} R_{i}: M^{t} R_{i}\right)_{R_{i}} / a_{i+1} R_{i}
$$

for sufficiently large $t>0$.

Let $n \geq i$. Given any $f_{n} X^{n} \in\left(a_{i+1} R_{i}: M^{t} R_{i}\right)_{R_{i}}, f_{n} \in \mathfrak{m}^{n} A_{i}$, we have

$$
f_{n} \in\left(a_{i+1} \mathfrak{m}^{n+t} A_{i}: \mathfrak{m}^{t} A_{i}\right) \subset\left(a_{i+1} A_{i}: \mathfrak{m}^{t} A_{i}\right) \cap\left(\mathfrak{m}^{n+t+1} A_{i}: \mathfrak{m}^{t} A_{i}\right) .
$$

Suppose $i \leq d-2$. Then

$$
\left(a_{i+1} A_{i}: \mathfrak{m}^{t} A_{i}\right)=U\left(a_{i+1} A_{i}\right) .
$$

On the other hand,

$$
\left(\mathfrak{m}^{n+t+1} A_{i}: \mathfrak{m}^{t} A_{i}\right) \subset\left(\mathfrak{m}^{n+t+1} A_{i}: a_{i+1}^{t} A_{i}\right) .
$$

By (2.6),

$$
f_{n} \in U\left(a_{i+1} A_{i}\right) \cap\left(\mathfrak{m}^{n+t+1} A_{i}: a_{i+1}^{t} A_{i}\right)=U\left(a_{i+1} A_{i}\right) \cap \mathfrak{m}^{n+1} A_{i}=a_{i+1} \mathfrak{m}^{n} A_{i} .
$$

Hence $f_{n} X^{n} \in a_{i+1} R_{i}$. Therefore

$$
\left[H_{M}^{0}\left(R_{i} / a_{i+1} R_{i}\right)\right]_{n}=(0) \text { for } n \notin[0, i-1] \text {. }
$$

Suppose $i=d-1$. Then we may choose $t$ so that $\left(a_{d} A_{d-1}: \mathfrak{m}^{t} A_{d-1}\right)=A_{d-1}$. By c), $\mathfrak{m}^{n+t+1} A_{d-1}=a_{d}^{t+1} \mathfrak{m}^{n} A_{d-1}$. 
Therefore

$$
f_{n} \in\left(a_{d}^{t+1} \mathfrak{m}^{n} A_{d-1}: a_{d}^{t} A_{d-1}\right)=a_{d} \mathfrak{m}^{n} A_{d-1} .
$$

Hence $f_{n} X^{n} \in a_{d} R_{d-1}$. Thus we have

$$
\left[H_{M}^{0}\left(R_{i} / a_{i+1} R_{i}\right)\right]_{n}=(0) \quad \text { for } 0 \leq i<d \text { and } n \notin[0, i-1] .
$$

Now we can complete the proof of Theorem (0.1) by the following proposition.

Proposition (2.11). $\left[H_{M}^{j}\left(R_{i}\right)\right]_{n}=(0)$ for $0 \leq i<d, 1 \leq j \leq d-i$ and $n \notin[0, i-1]$.

In fact, for $i=0$ we have $R_{0}=R$. Since [0, -1] is empty (2.11) shows that $H_{M}^{j}(R)=(0)$ for $1 \leq j \leq d$. On the other hand, $H_{M}^{0}(R)=(0)$ since $\operatorname{depth} A>0$. Hence $R$ is Cohen-Macaulay as required.

Proof of Proposition (2.11). We prove this by descending induction on $i$. For $i=d-1$, we know from (2.10) that

$$
\left[H_{M}^{0}\left(R_{d-1} / a_{d} R_{d-1}\right)\right]_{n}=(0)
$$

for $n \notin[0, d-2]$. From the exact sequence

$$
0 \longrightarrow R_{d-1} \stackrel{a_{d}}{\longrightarrow} R_{d-1} \longrightarrow R_{d-1} / a_{d} R_{d-1} \longrightarrow 0
$$

we obtain the exact sequence

$$
H_{M}^{0}\left(R_{d-1} / a_{d} R_{d-1}\right) \longrightarrow H_{M}^{1}\left(R_{d-1}\right) \stackrel{a_{d}}{\longrightarrow} H_{M}^{1}\left(R_{d-1}\right) .
$$

Hence $a_{d}$ is a non-zero divisor on $\left[H_{M}^{1}\left(R_{d-1}\right)\right]_{n}$ for $n \notin[0, d-2]$. For any $x \in\left[H_{M}^{1}\left(R_{d-1}\right)\right]_{n}, n \notin[0, d-2]$, there exist a positive integer $t$ such that $a_{d}^{t} x=0$. Hence $x=0$. Thus $\left[H_{M}^{1}\left(R_{d-1}\right)\right]_{n}=(0)$ for $n \notin[0, d-2]$. For $i<$ $d-1$,

$$
\left[H_{M}^{j}\left(R_{i+1}\right)\right]_{n}=(0) \quad \text { for } 1 \leq i \leq d-i-1 \text { and } n \notin[0, i]
$$

by induction hypothesis. By (2.7)

$$
{ }_{n} H_{\mathrm{m}}^{j}\left(U\left(a_{i+1} A_{i}\right)\right) \longrightarrow H_{M}^{j}\left(R_{i} / a_{i+1} X R_{i}\right) \longrightarrow H_{M}^{j}\left(R_{i+1}\right)
$$

is exact for $j>0$. Hence

(*) $\left[H_{M}^{j}\left(R_{i} / a_{i+1} X R_{i}\right)\right]_{n}=(0) \quad$ for $1 \leq j<d-i$ and $n \notin[0, i]$.

By (2.8)

$$
H_{M}^{j}\left(G_{i}\right)(-1) \longrightarrow H_{M}^{j}\left(R_{i} / a_{i+1} R_{i}\right) \longrightarrow H_{M}^{j}\left(R_{i+1}\right)
$$


is exact for $j>0$. Hence, by (2.4) and (2.9)

(**) $\quad\left[H_{M}^{j}\left(R_{i} / a_{i+1} R_{i}\right)\right]_{n}=(0) \quad$ for $1 \leq j<d-i$ and $n \notin[0, i]$.

From the exact sequence

$$
0 \longrightarrow R_{i} \stackrel{a_{i+1}}{\longrightarrow} R_{i} \longrightarrow R_{i} / a_{i+1} R_{i} \longrightarrow 0
$$

we get the exact sequence

$$
H_{M}^{j}\left(R_{i} / a_{i+1} R_{i}\right) \longrightarrow H_{M}^{j+1}\left(R_{i}\right) \stackrel{a_{i+1}}{\longrightarrow} H_{M}^{j+1}\left(R_{i}\right)
$$

for $j \geq 0$.

Hence $\left[H_{M}^{1}\left(R_{i}\right)\right]_{n}=(0)$ for $n \notin[0, i-1]$ by $(2.10)$. For $j \geq 2$, by $(* *)$ above

$(* * *) \quad\left[H_{M}^{j}\left(R_{i}\right)\right]_{n}=(0) \quad$ for $2 \leq j \leq d-i$ and $n \notin[0, i]$.

From the exact sequence

$$
0 \longrightarrow R_{i}(-1) \stackrel{a_{i+1} X}{\longrightarrow} R_{i} \longrightarrow R_{i} / a_{i+1} X R_{i} \longrightarrow 0
$$

we have the following exact sequence

$$
H_{M}^{j}\left(R_{i} / a_{i+1} X R_{i}\right) \longrightarrow H_{M}^{j+1}\left(R_{i}\right)(-1) \longrightarrow H_{M}^{j+1}\left(R_{i}\right)
$$

for $j \geq 0$. Hence, by $(*)$ and $(* * *)$

$$
\left[H_{M}^{j}\left(R_{i}\right)\right]_{n}=(0) \quad \text { for } 1 \leq j \leq d-i \text { and } n \notin[0, i-1] .
$$

This completes the proof of Proposition (2.11) and hence of Theorem (0.1).

Corollary (2.12) (S. Goto and Y. Shimoda). Let $(A, \mathfrak{m}, k)$ be a CohenMacaulay local ring of dimension $d>0$. Then $R(A)$ is Cohen-Macaulay if and only if $G(A)$ is Cohen-Macaulay and $\mathfrak{m}^{d}=\left(a_{1}, \cdots, a_{d}\right) \mathfrak{m}^{d-1}$ for some $a_{1}, \cdots, a_{d} \in \mathfrak{m}$.

Proof. This follows from the fact that $A$ is Cohen-Macaulay if and only if $H_{\mathrm{m}}^{i}(A)=(0)$ for $i<d$.

Remark. We have shown $\operatorname{depth} A>0$ if $R(A)$ is Cohen-Macaulay in the proof of Theorem (0.1). But more can be said about depth $A$. In fact, we can prove $\operatorname{depth} A \geq \min \{2, d\}$ if $R(A)$ is Cohen-Macaulay. A proof can be found in [2], but here we give a proof based on our result.

Proof. We have the following exact sequence

$$
0 \longrightarrow H_{\mathfrak{R}}^{0}(G) \longrightarrow H_{\Re}^{0}\left(G /\left(h_{1}\right)\right) \longrightarrow H_{\mathfrak{P}}^{1}(G)(-1) \longrightarrow 0 \text {. }
$$


But $H_{\mathfrak{\beta}}^{0}(G)=(0)$ by b). Hence $\left[H_{\Re}^{0}\left(G /\left(h_{1}\right)\right)\right]_{0}=H_{\mathrm{m}}^{1}(A)$ by the above exact sequence and $b)$. On the other hand, by the definition of local cohomology we have $\left[H_{\Re}^{0}\left(G /\left(h_{1}\right)\right)\right]_{0}=(0)$. Therefore $H_{\mathrm{m}}^{1}(A)=(0)$ as required.

\section{§3. Example}

In this section we construct a Buchsbaum ring which is not CohenMacaulay and whose Rees algebra is Cohen-Macaulay.

Let $k$ be a field and $d \geq 3$ be an integer and let $X_{1}, \cdots, X_{d}$ be indeterminates over $k$. We put $S=k\left[\left[X_{1}, \cdots, X_{d}\right]\right]$. Let

$$
0 \longrightarrow S \stackrel{f_{d}}{\longrightarrow} S^{a} \longrightarrow \cdots \stackrel{f_{3}}{\longrightarrow} \stackrel{2}{\wedge}^{\longrightarrow} S^{d} \stackrel{f_{2}}{\longrightarrow} S^{d} \stackrel{f_{1}}{\longrightarrow} S \longrightarrow 0
$$

be the Koszul complex with respect to $X_{1}, \cdots, X_{d}$. Let $E=\operatorname{Im} f_{2}$. Let $A=S \times E$ be the idealization of $E$. Then, it is easy to see that

$$
A \stackrel{\sim}{\sim} k\left[\left[X_{1}, \cdots, X_{d},\left\{Y_{i j} \mid 1 \leq i<j \leq d\right\}\right]\right] / \mathfrak{a},
$$

where $Y_{i j}(1 \leq i<j \leq d)$ is an indeterminate over $S$ and $a$ is the ideal generated by

$$
X_{i_{1}} Y_{i_{2} i_{3}}-X_{i_{2}} Y_{i_{1} i_{3}}+X_{i_{3}} Y_{i_{1} i_{2}}\left(1 \leq i_{1}<i_{2}<i_{3} \leq d\right)
$$

and

$$
\left(\left\{Y_{i j} \mid 1 \leq i<j \leq d\right\}\right)^{2} .
$$

Let $x_{i}, y_{i j}$ be the canonical images of $X_{i}, Y_{i j}$ in $A$ and $\mathfrak{m}$ be the maximal ideal of $A$. Then, by construction $\operatorname{dim} A=d$ and

$$
H_{\mathrm{m}}^{i}(A)= \begin{cases}k & (i=2) \\ (0) & (i \neq 2, d) .\end{cases}
$$

Hence $A$ is Buchsbaum by the following lemma.

Lemma 3.1. Let $(A, \mathfrak{m}, k)$ be a Noetherian local ring of dimension $d>$ $\operatorname{depth} A=t$. Suppose $\mathrm{m}_{\mathrm{m}}^{t}(A)=(0)$ and $H_{\mathrm{m}}^{i}(A)=(0)$ for $i \neq t, d$. Then $A$ is Buchsbaum.

Proof. See [11].

It is easy to see that $\mathrm{m}^{2}=\left(x_{1}, \cdots, x_{d}\right) \mathfrak{m}$. On the other hand, since $\mathfrak{a}$ is generated by homogeneous polynomials $G(A)$ is isomorphic to

$$
k\left[X_{1}, \cdots, X_{d},\left\{Y_{i j} \mid 1 \leq i<j \leq d\right\}\right] / \mathfrak{b},
$$


where $\mathfrak{b}$ is the ideal generated by the polynomials generating $\mathfrak{a}$. Hence it is easy to check that for $i<d$

$$
\left[H_{\mathfrak{P}}^{i}(G(A))\right]_{n}= \begin{cases}H_{\mathrm{m}}^{i}(A) & (n=-1) \\ (0) & (n \neq-1) .\end{cases}
$$

Hence $R(A)$ is Cohen-Macaulay, but $A$ is not Cohen-Macaulay.

\section{REFERENCES}

[1] M. Auslander and D. A. Buchsbaum, Codimension and multiplicity, Ann. of Math., 68 (1958), 625-657.

[2] S. Goto and Y. Shimoda, On the Rees algebras of Cohen-Macaulay local rings, Commutative algebra (analytic methods), Lect. Notes in Pure and Appl. Math., 68 (1982), 201-231.

[ 3 ] _ On Rees algebras over Buchsbaum rings, J. Math. Kyoto Univ., 20 (1980), 691-708.

[4] A. Grothendieck, Local cohomology, Lect. Notes in Math., 41, Springer Verlag, Heidelberg, 1967, $116 \mathrm{pp}$.

[5] J. Matijevic and P. Roberts, A conjecture of Nagata on graded Cohen-Macaulay rings, J. Math. Kyoto Univ., 14 (1974), 125-128.

[6] D. G. Northcott and D. Rees, Reductions of ideals in local rings, Proc. Camb. Phil. Soc., 50 (1954), 145-158.

[ 7 ] B. Renschuch, J. Stuckrad and W. Vogel, Weitere Bemerkungen zu einem Problem der Schnittheorie und uber ein Maß von A. Seidenberg für die Imperfektheit, J. Algebra, 37 (1975), 447-471.

[ 8 ] P. Schenzel, Applications of dualizing complexes to Buchsbaum rings, Adv. in Math., 44 (1980), 61-77.

[9 ] P. Schenzel, N. V. Trun gand N. T. Cuong, Verallgemeinerte Cohen-MacaulayModuln, Math. Nachr., 85 (1978), 57-73.

[10] J. Stuckrad and W. Vogel, Eine Verallgemeinerung der Cohen-Macaulay Ringe und Anwendungen auf ein Problem der Multiplzitätstheorie, J. Math. Kyoto Univ., 13 (1973), 513-528.

[11] — Toward a theory of Buchsbaum singularities, Amer. J. Math., 100 (1978), 727-746.

[12] G. Valla, Certain graded algebras are always Cohen-Macaulay, J. Algebra, 42 (1976), 537-548.

Department of Mathematics

Faculty of Science

Nagoya University

Chikusaku, Nagoya

464 Japan 